\title{
Kounis syndrome and systemic mastocytosis in a 52-year-old man having surgery
}

\author{
Marina Lerner MD, Raveen S. Pal MD, Rozita Borici-Mazi MD
}

— Cite as: CMAJ 2017 February 6;189:E208-11. doi: 10.1503/cmaj.151314

A 52-year-old man was admitted to a community hospital for an elective resection of a lipoma $(2 \mathrm{~cm} \times 2 \mathrm{~cm})$ on his right chest wall for cosmetic reasons, which was to be done with local anesthetic only. Preoperatively, he received intravenous cefazolin. The site of the lipoma was infiltrated with a solution of bupivacaine hydrochloride and lidocaine hydrochloride. Generalized pruritus and flushing immediately developed and was treated with intravenous diphenhydramine. However, the patient became increasingly agitated, hypotensive and hypoxic, which necessitated sedation and intubation. Cardiac monitoring showed ST-segment elevation in the inferior leads followed by ventricular fibrillation and cardiac arrest. The patient received cardiopulmonary resuscitation, a total of seven defibrillations and $3 \mathrm{mg}$ of epinephrine intravenously. Return of circulation was achieved, and STsegment elevation seen on the electrocardiogram (ECG) resolved within minutes of epinephrine administration (Figure 1). Intravenous amiodarone hydrochloride was started and, within the next hour, the patient experienced self-limited episodes of bradycardia and long QT syndrome. The planned procedure was abandoned, and the patient was transferred to a tertiary care site for urgent angiography. He had an initial blood troponin T level of $0.17 \mu \mathrm{g} / \mathrm{L}$, with a maximum elevated peak level of $0.783 \mu \mathrm{g} / \mathrm{L}$.

Coronary catheterization showed minimal coronary artery disease, and no lesions seen that were consistent with ST-segment elevation myocardial infarction (STEMI) (Figure 2). An ECG showed a left ventricular ejection fraction of $70 \%$, with no wall motion abnormalities. As a result, we made a diagnosis of type I Kounis syndrome. The allergy service was consulted; however, a detailed history could not be obtained owing to the patient's confusion from anoxic brain injury. His serum tryptase level was substantially elevated $(29.3 \mathrm{ng} / \mathrm{mL}$; normal $3.8-11.4 \mathrm{ng} / \mathrm{mL}$ ) one week after the event.

Two months later, the patient's cognition had improved, and he provided a history that included long-standing recurring symptoms that were unexplained. He reported one episode of a decreased level of consciousness that was associated with urticaria, abdominal pain and diarrhea and had no known trigger. He also reported numerous episodes of feeling faint, generalized flushing and skin pruritus that led to repeated assessments at the emergency department. In addition, he had experienced three episodes of severe abdominal pain and diarrhea with associated flushing and wheezing, which led to three exploratory laparotomies that did not determine a cause.

\section{KEY POINTS}

- Cardiovascular involvement (e.g., chest pain, arrhythmias or abnormal electrocardiogram findings with normal or minimally elevated troponin levels) during an anaphylactic event should raise suspicion of Kounis syndrome.

- Management of Kounis syndrome is complex and should involve cardiology and allergy subspecialty services.

- Although epinephrine has been associated with uncommon adverse events in patients with Kounis syndrome, it is the drug of choice for anaphylaxis and should be considered for use in these patients after analysis of the risk:benefit ratio.

- Perioperative anaphylaxis may unmask underlying systemic mastocytosis.

The patient's past medical history included obstructive sleep apnea, hypertension, dyslipidemia and rhinitis. He also reported local reactions to insect stings and a oxytetracycline allergy. His medication list included diltiazem, budesonide nasal spray, acetylsalicylic acid (ASA) and atorvastatin. We suspected an underlying condition of systemic mastocytosis, and we conducted further investigations to confirm the diagnosis and presence of systemic involvement. The patient declined bone marrow testing and skin testing to local anesthetics and cephalosporins. A peripheral blood sample was used to conduct genetic testing, which showed a c-kit somatic mutation in codon 816 , pathognomic for systemic mastocytosis. Test results for specific immunoglobulin $\mathrm{E}$ (IgE) antibodies for latex and for a few cross-reacting antibiotics (e.g., amoxycilloyl, penicilloyl G, penicilloyl $\mathrm{V}$ and ampicilloyl) were negative. A pan-computed tomographic (CT) scan showed no apparent masses or organ involvement. A repeat test of serum tryptase showed that the level was still elevated $(31.2 \mathrm{ng} / \mathrm{mL})$. The patient was given a diagnosis of perioperative type I Kounis syndrome with underlying systemic mastocytosis; however, we could not rule out a drug allergy.

The patient was educated on the management of anaphylaxis and was prescribed epinephrine autoinjectors. For prophylactic treatment, he was started on cetirizine, ranitidine, montelukast and ketotifen. He had regular follow-ups at the allergy clinic for three years and his symptoms remained controlled, except for one spontaneous systemic reaction requiring epinephrine and chronically elevated tryptase levels that recently rose to $45.0 \mu \mathrm{g} / \mathrm{L}$. 


\section{Discussion}

Anaphylaxis is a severe, potentially fatal, acute systemic allergic reaction that occurs suddenly after contact with an allergen or spontaneously. In a few instances, anaphylaxis may manifest as Kounis syndrome. ${ }^{1,2}$ Kounis syndrome is defined as the concurrence of an acute coronary syndrome (e.g., coronary spasm, acute myocardial infarction or stent thrombosis) in association with mast cell activation. ${ }^{1,2}$ Mast cell activation is usually seen following an allergic insult but may be spontaneous, such as in mast cell activation syndromes. ${ }^{1-4}$ Kounis syndrome presents challenges with prompt recognition and simultaneous management of concurring events (i.e., anaphylaxis and acute coronary syndrome).

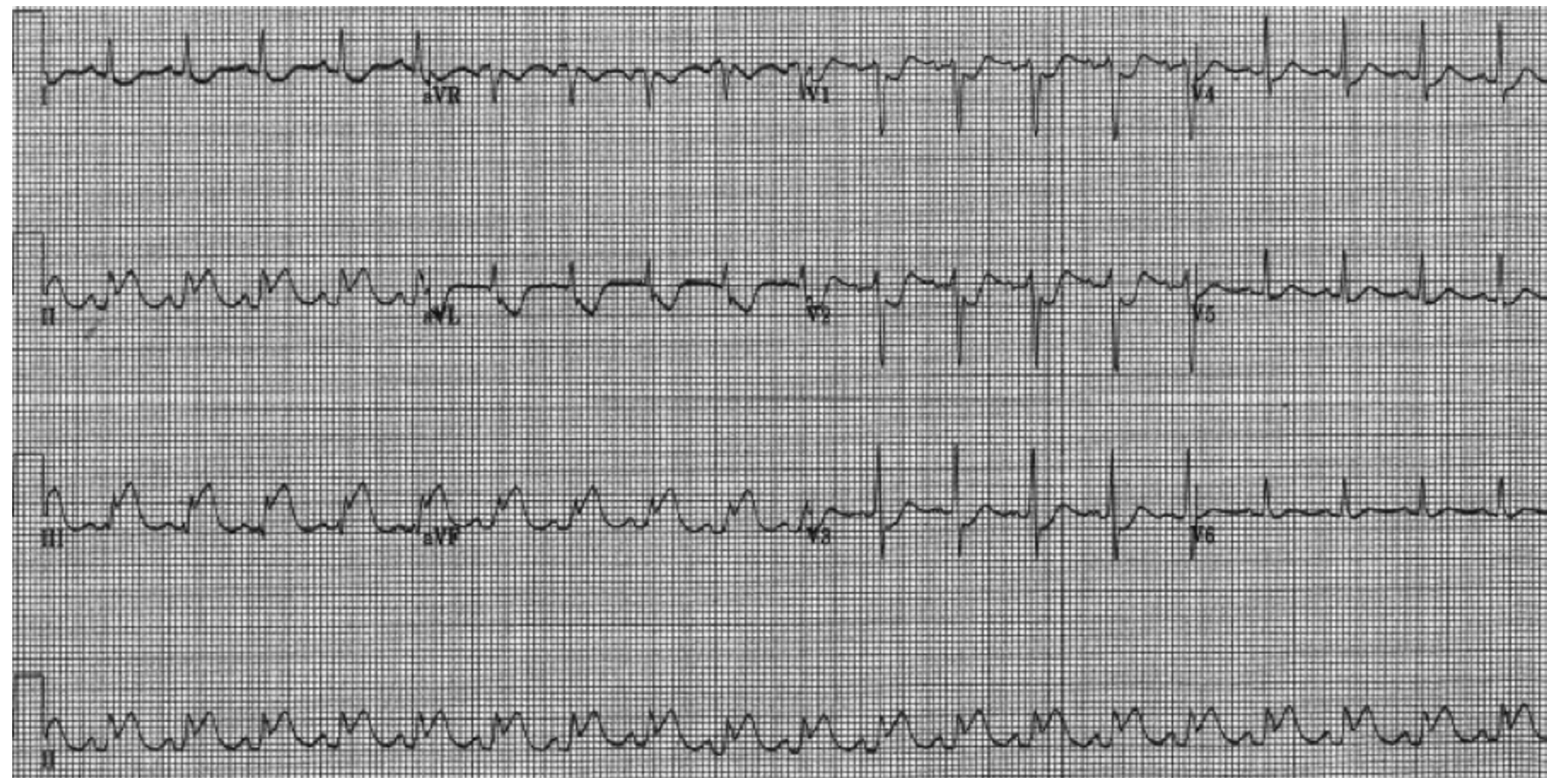

Figure 1: Electrocardiogram of a 52-year-old man with perioperative Kounis syndrome and systemic mastocytosis showing ST-segment elevation in the inferior leads (II, III and AvF) with reciprocal changes in I and AvL, which is consistent with ST-segment elevation myocardial infarction.

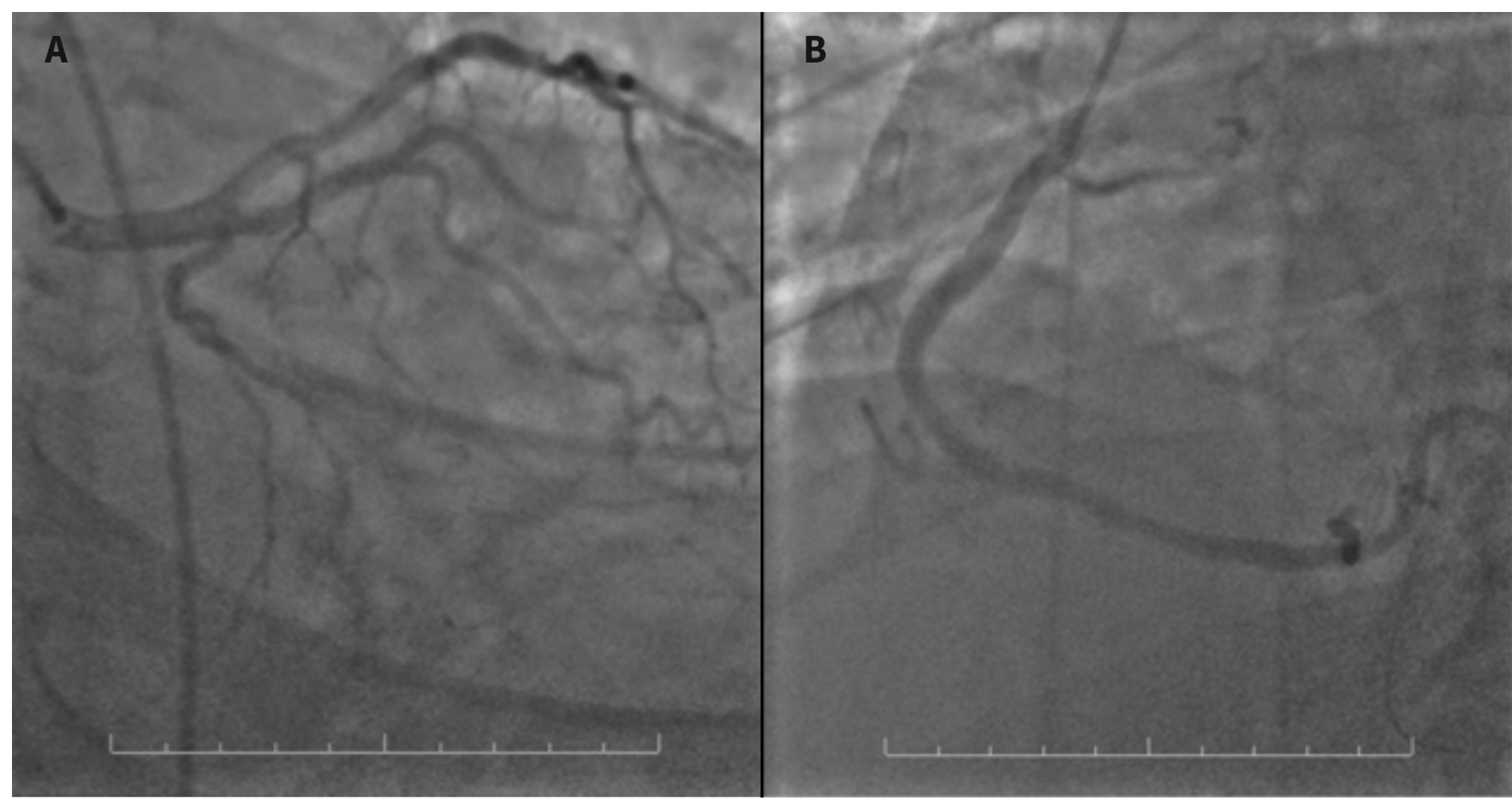

Figure 2: Coronary catheterization images (scale $10 \mathrm{~cm}$ ) showing $(A)$ circulation of the left coronary artery with minor disease and $(B)$ circulation in the right coronary artery with minor disease. 


\section{Recognition of Kounis syndrome}

Evaluation of perioperative anaphylaxis requires a thorough history, including a comprehensive list of medications used and sequence of events. In this case, the patient had sudden-onset generalized pruritus and flushing, followed by respiratory distress and unstable vital signs after receiving intravenous cefazolin and a local anesthetic. His symptoms indicated multisystem involvement, which is consistent with an anaphylactic reaction. However, given the rapid sequence of events, the STEMI observed upon ECG and ventricular fibrillation leading to cardiac arrest, we initially considered the possibility of an isolated acute coronary event, and the patient was managed accordingly. Eventually, we diagnosed Kounis syndrome based on evidence of cardiac involvement during anaphylaxis. Short-lived ECG findings, lack of a sufficient rise in troponin level and absence of a coronary lesion responsible for ST-segment elevation indicated coronary vasospasm, and given the the analphylatic reaction seen, the mechanism was likely allergic (Box 1)., ${ }^{1,2}$

\section{Evaluation}

Common causes of perioperative anaphylaxis include anesthetic agents, antibiotics, latex and colloidal fluids. Perioperative, spontaneous mast cell degranulation causing anaphylaxis has also been reported. ${ }^{5}$ Mastocytosis is a group of disorders characterized by clonal proliferation of abnormal spindle-shaped mast cells that accumulate in tissues and spontaneously release mediators. This triggers spontaneous anaphylactic reactions in response to nonallergic stimuli such as stress, medications and medical or surgical procedures. ${ }^{6}$

Systemic mastocytosis is an uncommon condition. The yearly incidence is $5-10$ new cases per million population. ${ }^{6}$ In most cases of systemic mastocytosis, the clonal nature of the disease can be established if a somatic A to T mutation of the coding sequence in the KIT gene is found. The resulting substitution of aspartate (D) to valine (V) at position 816 in the kinase domain leads to autoactivation of the KIT receptor tyrosine kinase, resulting in autonomous mast cell proliferation. ${ }^{6}$ An elevated tryptase level, obtained between 15 minutes and 3 hours of the start of symptoms, serves as a marker of release of mast cellderived mediators. A chronically elevated tryptase level (i.e., $>20 \mathrm{ng} / \mathrm{mL}$ ) is predictive of systemic mastocytosis. ${ }^{7}$

Our patient's tryptase level was substantially elevated one week after the event (when he was asymptomatic), which raised suspicion of underlying systemic mastocytosis. A long history of events suggestive of spontaneous mast cell degranulation and resolution of these symptoms after institution of prophylactic treatment for systemic mastocytosis also supported the clinical diagnosis of systemic mastocytosis.

Although the patient declined a confirmatory bone marrow test for aggregates of abnormal mast cells and skin testing to rule out drug allergy, we found somatic mutation of $816 \mathrm{c}-\mathrm{kit}$ in a peripheral blood sample using flow cytometry. This finding is highly suggestive of underlying systemic mastocytosis. ${ }^{7}$ Test results for specific IgE antibodies for latex and cross-reactive antibiotics were negative; however, the patient was advised to avoid these triggers, as latex or drug allergies could not be ruled out.

\section{Box 1: Classification of Kounis syndrome}

Type I:

- Acute coronary syndrome is due to a reaction, with normal or nearly normal coronary arteries.

Type II:

- Acute coronary syndrome occurs in conjunction with nonobstructive pre-existing atheromatous disease. The release of inflammatory mediators may cause vasospasm, plaque erosion or rupture.

Type III:

- Acute coronary syndrome occurs in patients with thrombosis in a drug-eluting coronary stent. The sample of the thrombus usually contains eosinophils and mast cells.

We concluded that, although the cause of Kounis syndrome remained unknown, a flare of underlying systemic mastocytosis caused by stress related to the surgical procedure likely contributed to the severity of anaphylactic reaction in our patient.

\section{Management}

\section{Kounis syndrome}

We conducted a literature review, but we did not find any guidelines for the management of Kounis syndrome, and our suggestions for management outlined below have been derived from review papers and case reports. Management of this syndrome is complex and should involve cardiology and allergy subspecialty services.

During anaphylaxis, the heart can be the source and the target of mast cell-derived mediators. ${ }^{1,2,8}$ Although epinephrine is well known as the treatment of choice for anaphylaxis, it has been suggested that epinephrine may aggravate ischemia and precipitate arrhythmias in patients with Kounis syndrome..$^{1,2,8} \mathrm{In}$ these patients, degranulated mast cells release histamine and a cascade of mediators that can provoke coronary arterial spasm, induce plaque rupture, increase myocardial contractility and enhance thrombus formation via platelet activation. Early intramuscular administration of epinephrine stabilizes mast cells and restores coronary blood flow in patients with cardiac involvement (e.g., chest pain, arrhythmias, abnormal ECG findings), whereas uncommon adverse events related to epinephrine use (e.g., ventricular arrhythmias or worsened coronary spasm) appear more likely to be caused by intravenous administration. ${ }^{9}$ In this case, the patient had ventricular fibrillation and cardiac arrest during anaphylactic reaction; epinephrine, although administered intravenously, contributed to successful resuscitation.

For patients with Kounis syndrome, we suggest a careful analysis of individual risks and benefits when considering using epinephrine, an approach that is consistent with the conclusion in a 2015 review on anaphylaxis and cardiac disease that "concerns about potential adverse effects related to epinephrine use need to be weighed against concerns about possible death from untreated anaphylaxis, but there is no absolute contraindication

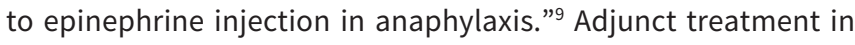


anaphylaxis using intravenous fluids, combination antihistamines, inhaled $\beta$-agonists and steroids has not been shown to interfere with management of concurrent cardiac events., ${ }^{8,9}$

Initial management of acute coronary syndromes includes antiplatelet therapy, anticoagulation therapy, pain control and $\beta$-blockade. ${ }^{10}$ However, some of these agents should be used with caution in the setting of Kounis syndrome because of the potential worsening anaphylactic symptoms. Although ASA is the first line antiplatelet treatment in patients with acute coronary syndrome, it should be avoided in patients with Kounis syndrome. ASA may shunt the breakdown of arachidonic acid into the leukotriene pathway, producing more leukotrienes that are mediators of anaphylaxis. ${ }^{1,2,8}$ Morphine is another commonly used medication to control chest pain in acute coronary syndrome. ${ }^{10}$ Morphine may induce nonspecific mast cell degranulation and should also be avoided. Fentanyl and its derivatives show only a slight activation of mast cells and would be a better choice when narcotic analgesia is necessary. ${ }^{1,2,8}$ Finally, $\beta$-blockers should be used with caution, because they may offset the benefits of epinephrine in managing anaphylaxis. Furthermore, glucagon should be considered for treatment of anaphylaxis in patients who have received $\beta$-blocking agents. ${ }^{1,2,8}$

\section{Systemic mastocytosis}

Management of patients with systemic mastocytosis includes education about prompt recognition and treatment of anaphylaxis. Medications used in prophylaxis of anaphylactic episodes include combinations of $\mathrm{H}_{1}$ - and $\mathrm{H}_{2}$-antihistamines, leukotriene antagonists, mast cell stabilizers and, on occasion, corticosteroids. ${ }^{6}$ Patients with systemic mastocytosis should be made aware of various exposures and situations that can spontaneously trigger symptoms. ${ }^{5-7}$ These include insect stings, surgery or endoscopic procedures, anesthesia, alcohol, spicy food, infection, vaccination, strenuous exercise, acute emotional stress and exposure to cold or heat. ${ }^{5-7}$ Use of premedication before surgical and endoscopic procedures is recommended for these patients. ${ }^{5}$

\section{Conclusion}

Kounis syndrome is well described, although it is not a commonly reported phenomenon perioperatively. Prompt recognition of cardiac involvement during anaphylaxis, identification of the cause and consideration of possible underlying systemic mastocytosis are important in the management of Kounis syndrome.

\section{References}

1. Kounis NG, Zavras GM. Histamine-induced coronary artery spasm: the concept of allergic angina. Br J Clin Pract 1991;45:121-8.

2. Kounis NG. Coronary hypersensitivity disorder: the Kounis syndrome. Clin Ther 2013;35:563-71.

3. Lleonart R, Andres B, Makatsori M, et al. Systemic mastocytosis presenting as Kounis syndrome. Ann Allergy Asthma Immunol 2013;111:570-1.

4. González-de-Olano D, Matito A, Sánchez-López P, et al. Mast cell-related disorders presenting with Kounis syndrome. Int J Cardiol 2012;161:56-8.

5. Mertes PM, Alla F, Tréchot $\mathrm{P}$, et al. Anaphylaxis during anesthesia in France: an 8-year national survey. J Allergy Clin Immunol 2011;128:366-73.

6. Magliacane D, Parente R, Triggiani M. Current concepts on diagnosis and treatment of mastocytosis. Transl Med UniSa 2014;8:65-74.

7. Akin C, Valent P. Diagnostic criteria and classification of mastocytosis in 2014 Immunol Allergy Clin North Am 2014;34:207-18.

8. Cevik C, Nugent K, Shome K, et al. Treatment of Kounis syndrome. Int J Cardiol 2010;143:223-6.

9. Lieberman $P$, Simons FER. Anaphylaxis and cardiovascular disease: therapeutic dilemmas. Clin Exp Allergy 2015;45:1288-95.

10. Fitchett $\mathrm{DH}$, Theroux $\mathrm{P}$, Brophy JM, et al. Assessment and management of acute coronary syndromes (ACS): a Canadian perspective on current guidelinerecommended treatment - part 2: ST-segment elevation myocardial infarction. Can J Cardiol 2011;27 Suppl A:S402-12.

\section{Competing interests: None declared.}

This article has been peer reviewed.

The authors have obtained patient consent.

Affiliations: Division of Respirology (Lerner), Department of Medicine, McMaster University, Hamilton, Ont.; Department of Medicine (Pal, Borici-Mazi), Queens University, Kingston, Ont.
Contributors: Rozita Borici-Mazi contributed to the concept and design of the manuscript. Marina Lerner was responsible for acquisition and interpretation of the data, drafted the manuscript and participated in subsequent revisions. Raveen Pal and Rozita Borici-Mazi contributed to the acquisition and interpretation of the data, and revised the manuscript critically for intellectual content. All of the authors gave final approval of the version to be published and agreed to act as guarantors of the work.

Correspondence to: Rozita Borici-Mazi, rb62@queensu.ca 